Running head: TACTILE CHANGE DETECTION DURING BODILY THREAT

\title{
DETECTION OF TACTILE CHANGE ON A BODILY LOCATION WHERE PAIN IS EXPECTED ${ }^{1}$
}

LORE VAN HULLE, WOUTER DURNEZ, GEERT CROMBEZ, \& STEFAAN VAN DAMME

Ghent University, Department of Experimental-Clinical and Health Psychology, Ghent, Belgium

\footnotetext{
${ }^{1}$ Address correspondence to Stefaan Van Damme, Ghent University, Department of Experimental-Clinical and Health Psychology, Henri Dunantlaan 2, 9000 Ghent, Belgium (e-mail: stefaan.vandamme@ugent.be)
} 
Summary. - As it is evolutionarily adaptive to accurately detect and localize bodily threats, it has been proposed that our brain prioritizes somatosensory input at body locations where pain is expected. To test this proposition, we investigated whether threat of pain facilitated the detection of tactile changes on the threatened body location. Healthy participants $(N=47)$ indicated whether two consecutive patterns of three tactile stimuli were the same or not. Stimuli could be administered at eight possible locations. In half of the trials, the same pattern was presented twice. In the other half, one stimulus location was different between the two displays. To induce bodily threat, a painful stimulus was occasionally administered to the non-dominant lower arm. Mean accuracy of tactile change detection as a function of location was analyzed using repeated measures ANOVA. We found that tactile changes at the threatened arm (i.e., when a tactile stimulus emerged at or disappeared from that arm), both at the exact pain location (lower arm) and at the other location (upper arm), were better detected than tactile changes at other limbs., 
Accurate detection and localization of pain and bodily threats is an evolutionarily adaptive ability, allowing protection of the body against actual or potential damage by triggering defensive behaviors (Dowman \& ben-Avraham, 2008; Eccleston \& Crombez, 1999; Haggard, Iannetti, \& Longo, 2013; Van Damme \& Legrain, 2012). Attention is believed to support this ability by prioritizing threat-relevant information (Legrain, Van Damme, Eccleston, Davis, Seminowicz, \& Crombez, 2009; Van Damme, Legrain, Vogt, \& Crombez, 2010). More specifically, it has been proposed that the brain possesses a multisensory salience detection system that orients and monitors attention to stimuli potentially threatening the integrity of the body (Legrain, Iannetti, Plaghki, \& Mouraux, 2011; Moseley, Gallace, \& Spence, 2012). Somatosensory changes at a pain-relevant bodily location may be prototypical signals for bodily threat, and therefore receive processing priority, resulting in better detection of these changes.

The empirical evidence for this intriguing idea is surprisingly scarce. However, Vanden Bulcke, Van Damme, Durnez, and Crombez (2013) attempted to address this issue. They had participants make temporal order judgments of pairs of tactile stimuli presented on the left and the right hands. Occasionally, a painful stimulus was also administered on one hand to induce bodily threat. They found that temporal order judgments were biased to the hand where pain was expected, i.e., tactile stimuli on the threatened hand were perceived earlier in time than stimuli on the other hand (Cohen's $d=0.70$; medium effect size). Although this finding suggests that somatosensory stimuli at a threatened bodily location are prioritized, it has to be noted that processing priority in that study was only operationalized as perceiving tactile stimuli at a threatened arm earlier in time than stimuli at the other arm. It is important to look also at other forms of prioritization, for exemple improved somatosensory perception at a threatened body location. Furthermore, studies are needed in which more than two body locations are involved, as this better resembles real life situations. 
The present study was designed to extend previous research findings, by assessing whether the threat of impending pain on a specific location of the body improves the detection of tactile changes on that particular body location. Undergraduate students performed a tactile change detection task where two consecutive patterns of innocuous tactile stimuli were presented on three out of eight possible body locations. In half of the trials, the same pattern was presented twice. In the other half of the trials, one stimulus location was different between the two displays. The participants had to judge whether the two patterns were the same or not. Previous studies have demonstrated that changes in tactile patterns often are not noticed (Gallace, Tan, \& Spence, 2006), but that focusing attention to one specific location increases the chance that tactile changes occurring there (a tactile stimulus emerging at or disappearing from that location) are detected (Van Hulle, Van Damme, Spence, Crombez, \& Gallace, 2013). In the present study, a painful stimulus was occasionally administered to the non-dominant lower arm, in order to experimentally induce bodily threat at that location. We hypothesized that this would result in heightened attention to the threatened body location, and thereby increase the chance that tactile changes occurring at this location would be detected.

\section{Method}

\section{Participants}

Forty-seven undergraduate psychology students ( 37 females, 10 males; mean age $=19.2$ years, range 17-28 years) took part in the experiment in order to fulfil course requirements. The study protocol was approved by the local ethical committee and was performed according to the ethical standards laid down in the declaration of Helsinki. The participants were informed that the experiment consisted of a computer-controlled task in which tactile stimuli would be administered to the arms and legs, and that painful (but harmless) electrocutaneous 
stimuli (ES) would be administered during this task. All participants provided informed consent and were free to terminate the experiment at any time should they so desire. All participants reported normal tactile perception at all tactor locations and normal or corrected to normal visual perception. Nine participants were excluded because technical problems led to a faulty administration of the ES.

\section{Apparatus and Materials}

The tactile stimuli $(200 \mathrm{~ms})$ were presented by means of eight resonant-type tactors (C-2 TACTOR, Engineering Acoustics, Inc., Florida) consisting of a housing that was 3.05 $\mathrm{cm}$ in diameter and $0.79 \mathrm{~cm}$ high, with a skin contactor that was $0.76 \mathrm{~cm}$ in diameter. All tactile stimulus characteristics (amplitude and frequency) were controlled by means of a selfdeveloped software program. The stimuli were administered to the dorsal aspects of eight different body locations (see Figure 1). These locations included the left and right lower arms, the left and right upper arms, the area just above the left and right ankles, and the area just below the left and right knees. The tactors were attached directly to the skin surface by means of double-sided tape rings and were driven by a custom-built device at $200 \mathrm{~Hz}$. Participants wore noise-cancelling headphones (PXC 350 Sennheiser) in order to prevent any interference from environmental noise. Prior to the start of the experiment, the perceived stimulus intensities at each tactor location were individually matched, as there is evidence for variation in sensitivity depending on the body site stimulated (e.g., Weinstein, 1968). In order to accomplish this, a standardized matching procedure, i.e. an non-automatized adaptive staircase procedure, was used for each participant (as in Van Hulle et al., 2013). First, a tactile stimulus (reference stimulus, Power $=0.04$ watts) was presented at the left lower arm. Next, a tactile stimulus was presented at one of the other body locations. Participants had to verbally report whether the intensity was lower than, higher than, or equal to the intensity of the reference stimulus. If the participant reported that the intensity of the latter stimulus was 
lower than the intensity of the reference stimulus, the step-size was increased by one; if the participant indicated that the intensity of the latter stimulus was higher than the intensity of the reference stimulus, the step-size was decreased by one. The staircase ended when the participant reported that the subjective intensity of each stimulus was perceived as being equal to the subjective intensity of the reference stimulus. This procedure was done separately for each tactor location. The reference stimulus was presented repeatedly before moving to another tactor location, in order to make sure that participants remembered the intensity of the reference stimulus correctly. Table 1 gives an overview of the mean objective intensities (Power, in watts) for each tactor location.

\section{INSERT TABLE 1}

The painful ES (bipolar; $3 \mathrm{~mA} ; 50 \mathrm{~Hz} ; 200 \mathrm{~ms}$; instantaneous rise and fall time) were delivered by means of a Constant Current Stimulator (DS5, Digitimer Ltd, Hertfordshire, UK) with two lubricated Medcat surface electrodes $(1 \mathrm{~cm}$ diameter). There was an acquaintance phase in which participants received a series of three stimuli of increasing intensities (respectively $1 \mathrm{~mA}, 2 \mathrm{~mA}$ and $3 \mathrm{~mA}$ ). The intensity of the last stimulus was effectively used in the experiment.

\section{The Tactile Change Detection Task}

The tactile change detection task (see also Van Hulle et al., 2013) was programmed and controlled by Inquisit Millisecond software (Inquisit 2.0) on a PC laptop (HP Compaq nc6120) with a keyboard. The participants were instructed to keep their eyes on the blackcoloured screen for the duration of the experiment. Each trial started with a white fixation cross that appeared in the center of the screen for $500 \mathrm{~ms}$. Next, the first tactile pattern was presented for $200 \mathrm{~ms}$, followed by an empty stimulus interval of $110 \mathrm{~ms}$, after which the 
second tactile pattern was presented for $200 \mathrm{~ms}$. Tactile patterns always consisted of three simultaneously presented tactile stimuli. Previous studies have shown that this provides the optimal performance level to allow detection of effects of experimental manipulation of attention (Gallace et al., 2006; Van Hulle et al., 2013). The different possible pattern combinations were randomly presented during the experiment. In half of the trials, the second pattern was identical to the first. In the other half of the trials, the two patterns differed, as one of the stimulated locations of the first tactile pattern shifted toward another location in the second tactile pattern. So, one of the three tactors that were active during the first pattern was inactive during the second pattern, and a tactor positioned at another body location became active instead. Examples of trials are provided in Figure 1. The participants were instructed to detect whether the first and the second tactile pattern differed, and to respond 'yes' or 'no' by pressing the corresponding response keys (respectively " 4 " and "6" on an AZERTYkeyboard) with the index and middle finger of their dominant hand. There was $2500 \mathrm{~ms}$ response time, and it was stressed that accuracy, rather than speed, was of importance.

\section{INSERT FIGURE 1}

\section{Procedure and Threat Manipulation}

In the acquaintance phase, a series of three ES's of increasing intensity was administered. Participants were asked to rate the painfulness and unpleasantness of the last ES on an 11-point rating scale ranging from 0 ("not at all") to 10 ("very much").

Before engaging in the tactile change detection task, the participants were informed that each trial consisted of the presentation of two tactile patterns that could either be identical or not. They were instructed to indicate whether the patterns were the same or not. As a manipulation of bodily threat, one of the stimulus locations was made threatening by 
informing the participants that during the change detection task, a painful ES could be administered to their non-dominant lower arm (i.e., adjacent to the tactor at that location). In $14.3 \%$ of the trials, a painful ES was actually administered to the threatened location.

Several types of trials are distinguished. In ES trials, an ES was presented on the nondominant forearm instead of either the first or the second tactile pattern. This temporal unpredictability was installed to avoid participants interpreting the administration of the first tactile pattern as a 'safety signal'. Participants were instructed not to respond to ES trials. In Same trials, the first and the second tactile pattern were identical. Of particular relevance for the hypothesis were the Change trials, which were divided into three categories, reflecting the relative position of the tactile change with regard to the threat location:

(1) Changes involving the exact threat location (THREATENED LOCATION). In these trials, the difference between the first and the second pattern involved the exact threat location (i.e., the lower part of the non-dominant arm). This means that after the first tactile pattern, a tactile stimulus was either added to the threat location, or omitted from that location. However, no actual ES was administered in these trials.

(2) Changes not involving the exact threat location, but on the same body part (THREATENED BODY PART). In these trials, the difference between the first and the second pattern did not involve the exact threat location, but another location on the same body part (i.e., the upper part of the non-dominant arm). These trials were included to test whether potential threat effects were specific for the threatened location, or were resulting from attentional prioritization of the whole body part.

(3) Changes not involving the threatened body part (NEUTRAL BODY PART). In these trials, the difference between the first and the second pattern involved one of the (notthreatened) locations on the other body parts (dominant arm, both legs). The threat 
location could be included in both patterns, but a tactile change never involved that location.

All tactor locations, including the threatened location, were stimulated an equal amount of times, namely in $37.50 \%$ of the same trials, change trials and ES trials. In the change trials, all tactor locations, including the threatened location, were involved in an equal amount $(12.50 \%)$ of the changes. A re-location of one tactile stimulus to another body location could occur from all body locations. The different trial types were presented randomly throughout the course of the experiment.

In order to become familiar with the task, the participants first performed a practice phase, consisting of 16 trials. In the experimental phase, the participants completed a total of 448 trials, divided into four blocks of 112 trials (16 ES trials, 48 same trials, 48 change trials). The ES trials were not analyzed, as in these trials one of the tactile patterns was replaced by an ES.

After the experiment, participants were asked to complete a number of self-reports assessing the experienced painfulness and unpleasantness of the ES that was administered during the experiment, to what extent they expected that a painful ES would be administered during the experiment, fear for the painful ES, anxiety during the experiment, and to what extent they attended to the threatened body location. Participants were asked to rate these items on an 11-point scale ranging from 0 ("not at all”) to 10 ("very much").

\section{Statistical Analysis}

The data were analyzed using repeated measures Analyses of Variance (ANOVAs), with location ('threat location involved', 'threatened limb involved but not threat location', 'threatened limb not involved') as a within-subject factor. Only the Change trials were included in the analyses in order to test our hypothesis. To obtain an objective and standardized measure of the magnitude of the observed effects, namely a standardized 
difference between two means, effect sizes (Cohen's $d$ ) for independent samples were calculated (Cohen, 1988). The 95\% Confidence Interval (95\% CI) was also calculated. Cohen's $d$ is an effect size that is not design-dependent and conventional norms are available (Field, 2005). We determined whether Cohen's $d$ was small (0.20), medium (0.50), or large (0.80). Given the medium effect size reported in Vanden Bulcke et al. (2013), we calculated that at least 34 participants were required to detect a similar effect with a power of $80 \%$.

\section{Results}

Analyses were performed on the data of 38 participants. Trials in which participants failed to give a response $(0.3 \%$ of the trials $)$ were not included the data analyses. The participants correctly responded to Same trials in $93.01 \%(S D=7.01)$ of these trials. On average, the participants correctly detected tactile changes in $68.66 \%(S D=16.40)$ of the Change trials.

\section{Tactile Change Detection}

A repeated measures ANOVA was performed on the proportion accurately detected change trials as the dependent variable and location ('threatened location', 'threatened body part', 'neutral body part') as the within-subjects factor in order to test the hypothesis that tactile changes would be better detected when the threat location was involved.

Figure 2 provides an illustration of the results. The main effect of location was significant $(F(2,36)=5.86, p<.01)$. Paired-samples $t$-tests indicated that the proportion of accurately detected tactile changes was significantly larger in 'threatened location' trials $(M=$ $0.71, S D=0.20)$ as compared to 'neutral body part' trials $(M=0.66, S D=0.17 ; t(1,37)=$ $2.18, p<.05 ; d=0.26,95 \%$ CI $[0.03,0.50])$, but not as compared to 'threatened body part' trials $(M=0.73, S D=0.18),(t(1,37)=1.05, n . s . ; d=-0.10,95 \%$ CI $[-0.36,0.15])$. In addition, the proportion of accurately detected changes was also significantly larger in 'threatened body 
part' trials than in 'neutral body part' trials $(t(1,37)=3.32, p<.01 ; d=0.40,95 \%$ CI $[0.13$, $0.67]$.

\section{INSERT FIGURE 2}

\section{Self-report Data}

The means, standard deviations and ranges of the self-report items that were administered before and after the experimental phase are in Table 2. Overall, participants reported that they experienced the ES as painful and unpleasant, and that they fearfully anticipated the administration of the ES. Note that participants' ratings of painfulness and unpleasantness do not significantly differ prior and after the experiment (respectively $t(45)=$ $1.16, p>.05$ and $t(45)=1.54, p>.05)$, indicating that there was no habituation during the experimental phase.

\section{INSERT TABLE 2}

\section{Discussion}

The present study investigated by means of a tactile change detection task (Gallace et al., 2006; Van Hulle et al., 2013) whether the threat of impending pain on a specific location of the body increases the chance that tactile changes on that particular body location, i.e., whether a tactile stimulus emerges or disappears at that location, are detected. Participants were instructed to detect changes between two consecutively presented patterns of tactile stimulation presented at different locations of the body. Pain expectation was experimentally induced by occasionally administering a painful stimulus at the non-dominant lower arm. Based on the idea of evolutionary development of a sensory salience detection system 
(Legrain et al., 2011), it was expected that this would install heightened attention to this location, resulting in a better detection of tactile changes involving that location.

The data partially supported our hypothesis. Tactile changes were indeed better detected when they occurred on the non-dominant lower arm, i.e., at the location that was threatened, as compared to locations at other body parts. However, also tactile changes at the non-dominant upper arm were better detected, and there was no significant difference in tactile change detection between the lower and upper non-dominant arm. These results suggest that the expectation of pain at a certain body location may have made participants more aware of tactile changes involving the whole body part on which pain was expected, rather than the exact threatened location.

Accurate detection and localization of bodily threats allows protection of the body against actual or potential damage by triggering defensive behaviors (Dowman \& benAvraham, 2008; Eccleston \& Crombez, 1999). Neurocognitive theories have proposed that the brain possesses a multisensory salience detection system that orients and monitors attention to stimuli potentially threatening the integrity of the body (Haggard et al., 2013; Legrain et al., 2011). We hypothesized that such protective mechanisms should lead to attentional prioritization of somatosensory changes at a threatened bodily location (Vanden Bulcke et al., 2013). While the data are in line with this idea, they also suggest that in a situation in which pain is expected, attentional prioritization may not be limited to the exact pain location. Rather, it seems that participants became more attentive to tactile changes at other locations of the same body part. This intriguing finding calls for an explanation. One could argue, for example, that the motor preparation possibly activated to avoid a potential painful stimulus on the lower arm requires both the lower and the upper arm. Consequently, tactile changes in the whole arm may have become more salient, and as such were more easily detected. In line with this reasoning, one may wonder whether this effect is limited to the 
same limb, or whether it is a pure distance effect. More research specifically testing the spatial boundaries of the attentional prioritization effect of pain anticipation on tactile perception is clearly needed. One reason why participants did not detect changes at the exact pain location better than tactile changes at another location on the same body part may be that the occasional administration of painful stimuli at the lower arm could have decreased the sensitivity for somatosensory information in that region. Such sensory gating effects (e.g., Apkarian, Stea, \& Bolanowski, 1994; Bolanowski, Maxfield, Gescheider, \& Apkarian, 2000) may have counteracted the possible enhancing effects of focused attention to the exact threat location. However, our results cannot confirm this thesis, as we did not compare tactile sensitivity before and after the experiment.

Our study of the effects of anticipated bodily threat on tactile processing at the threatened location should not be confused with studies investigating the effects of pain itself on tactile perception. A typical finding is that tactile thresholds on the hand are elevated by co-occurring, ipsilateral, tonic pain stimulation (e.g., Apkarian et al., 1994; Bolanowski et al., 2000). This phenomenon, often referred to as "touch gating", has been shown to be a purely sensory rather than a cognitive effect (Harper \& Hollins, 2012). One study has even found that when tactile stimulation is applied after pain (rather than simultaneously), processing of these stimuli in the somatosensory cortices is facilitated instead of attenuated (Ploner, Pollok, \& Schnitzler, 2004). The effect of touch gating is not expected to extend to anticipated pain, as the stimulus-locked sensory mechanisms activated by the presence of pain are absent. Instead, we expect the anticipation of pain to affect the processing of tactile stimuli at a cognitive level. Given the close correspondence between pain and touch, it may be assumed that tactile changes in a body region where pain is expected are particularly salient and will therefore receive processing priority. One might, for example, expect that in a situation in which a person is performing a potential pain-inducing movement, this person may scan the 
relevant limb in order to quickly detect potential threats. As a result, in the absence of actual pain, somatosensory information at the relevant body region may be processed thoroughly. However, as soon as pain is present, it may be more important to act upon this pain (Eccleston \& Crombez, 1999), as a result of which the processing of subtle somatosensory changes may become less relevant.

The present work may have relevance for clinical pain models and research. Cognitive-behavioral pain models have proposed that fearful appraisal and anticipation of pain enhances attention towards cues signaling potential pain or bodily harm, and that such "hypervigilance" may be particularly prominent in patients with chronic pain (Crombez, Van Damme, \& Eccleston, 2005; Vlaeyen \& Linton, 2000). The tactile detection paradigm, used in the present study, may be a valuable tool to investigate the idea of hypervigilance in clinical populations. Individuals with chronic low back pain or persistent orofacial pain, for example, may then be hypothesized to exhibit prioritized tactile attention at the specific region of the body where their pain problem is situated. Such research would complement previous studies investigating somatosensory sensitivity in patients with chronic pain (Geisser, Casey, Brucksch, Ribbens, Appleton, \& Crofford, 2003; Hollins, Harper, Gallagher, Owings, Lim, Miller, Siddiqi, \& Maixner, 2009; Peters, Vlaeyen, \& Kunnen, 2002).

Note that it is not clear in those studies whether increased somatosensory sensitivity was due to sensory abnormalities, attentional mechanisms, or a combination of both, and that sensitivity was measured on arbitrary body locations. One benefit of the current procedure is the fact that we controlled for potential confounds due to individual differences in tactile sensitivity. There is evidence that within an individual, sensitivity differs depending on the body part that is stimulated (Weinstein, 1968). In the current paradigm, the different stimulus intensities were matched prior to the experiment, as result of which the results cannot be attributed to sensory differences. The finding that pain anticipation facilitates the processing 
of somatosensory information in the corresponding body region may also be weighted against research showing that, in certain clinical samples such as complex regional pain syndrome and chronic low back pain, tactile perception in the affected region of the body is reduced (Moseley, 2008; Moseley, Gallagher, \& Gallace, 2012), suggesting a neglect-like phenomenon. Although these findings are, at first sight, in contrast with the idea of attentional prioritization of tactile stimuli at a threatened body part, it may be that the mechanisms underlying tactile processing in the context of clinical and experimental pain are fundamentally different. One challenge for future research is to investigate how these apparently opposing mechanisms are integrated in patients with chronic pain and if there are subtypes of patients that either prioritize or neglect tactile information at the affected body part.

There are a number of limitations to the present study. First, the threat location did not vary across or within subjects. As a result, it cannot fully be excluded that the participants' better performance in detecting changes occurring at the threatened limb may, for example, be due to participants being better able to detect changes at the arms as compared to the legs. Future research will need to disentangle this confound between body location and threat location. Second, we did not include a control condition in which the painful stimulus is replaced by a non-painful somatosensory stimulation, or even a stimulus from a different sensory modality. Such control condition would allow to examine if the effect is pain-specific, and also to exclude alternative explanations, such as higher accuracy being the mere result of a higher probability of sensory input at the threatened location. Third, no control condition was included with a non-somatic change detection task, as a result it is unclear if prioritization effects would also generalize to stimuli of other sensory modalities, as has previously been suggested (Mouraux, Diukova, Lee, Wise, \& Iannetti, 2011; Van Damme, Crombez, \& Lorenz, 2007). Fourth, the tactile change detection task only allows assessing accuracy in 
detecting changes in spatial configuration of tactile patterns. Our findings do not necessarily generalize to other forms of tactile changes, such as changes in intensity or other features of tactile stimuli. Fifth, painful stimuli were not individually calibrated, so it is possible that differences in perceived pain will have increased inter-individual variability in attention effects.

In sum, the present study suggests that the threat of impending pain on a specific location of the body leads to heightened attention to innocuous tactile information presented on that specific body location, and to the whole body part involving this threatened location. However, future research is needed to validate the current results and further investigate the scope of the attentional processing of tactile information under conditions of bodily threat. An interesting avenue for further research considers the study of attentional processing of somatosensory information in patients with chronic pain.

Acknowledgments

This research was funded by the Special Research Fund of Ghent University (BOF09/DOC/013 and BOF11/STA/004). 


\section{References}

Apkarian, A. V., Stea, R. A., \& Bolanowski, S. J. (1994) Heat-induced pain diminishes vibrotactile perception: A touch gate. Somatosensory \& Motor Research, 11, 259-267.

Bolanowski, S. J., Maxfield, L. M., Gescheider, G. A., \& Apkarian, A. V. (2000) The effects of stimulus location on the gating of touch by heat-and cold-induced pain. Somatosensory \& Motor Research, 17, 195-204.

Cohen, J. (1988) Statistical power analysis for the behavioral sciences. San Diego, CA: McGraw-Hill.

Crombez, G., Van Damme, S., \& Eccleston, C. (2005) Hypervigilance to pain: An experimental and clinical analysis. Pain, 116, 4-7.

Dowman, R., \& ben-Avraham, D. (2008) An artificial neural network model of orienting attention toward threatening somatosensory stimuli. Psychophysiology, 45, 229-239.

Eccleston, C., \& Crombez, G. (1999) Pain demands attention: A cognitive-affective model of the interruptive function of pain. Psychological Bulletin, 125, 356-366.

Field, A. (2005) Discovering statistics using SPSS (2 ${ }^{\text {nd }}$ edn.). London: Sage Publications

Gallace, A., Tan, H. Z., \& Spence, C. (2006) The failure to detect tactile change: A tactile analogue of visual change blindness. Psychonomic Bulletin \& Review, 13, 300-303

Geisser, M. E., Casey, K. L., Brucksch, C. B., Ribbens, C. M., Appleton, B. B., \& Crofford, L. J. (2003) Perception of noxious and innocuous heat stimulation among healthy women and women with fibromyalgia: Association with mood, somatic focus, and catastrophizing. Pain, 102, 243-250.

Haggard, P., Iannetti, G. D., \& Longo, M. R. (2013) Spatial sensory organization and body representation in pain perception. Current Biology, 23, R164-R176.

Harper, D. E., \& Hollins, M. (2012) Is touch gating due to sensory or cognitive interference? Pain, 153, 1082-1090. 
Hollins, M., Harper, D. E., Gallagher, S., Owings, E. W., Lim, P. F., Miller, V., Siddiqi, M. Q., \& Maixner, W. (2009) Perceived intensity and unpleasantness of cutaneous and auditory stimuli: An evaluation of the generalized hypervigilance hypothesis. Pain, $141,215-221$.

Legrain, V., Iannetti, G. D., Plaghki, L., \& Mouraux, A. (2011) The pain matrix reloaded: A salience detection system for the body. Progress in Neurobiology, 93, 111-124.

Legrain, V., Van Damme, S., Eccleston, C., Davis, K. D., Seminowicz, D. A., \& Crombez, G. (2009) A neurocognitive model of attention to pain: Behavioral and neuroimaging evidence. Pain, 144, 230-232.

Moseley, G. L. (2008) I can't find it! Distorted body image and tactile dysfunction in patients with chronic back pain. Pain, 140, 239-243.

Moseley, G. L., Gallace, A., \& Spence, C. (2012) Bodily illusions in health and disease: Physiological and clinical perspectives and the concept of a cortical 'body matrix'. Neuroscience \& Biobehavioral Reviews, 36, 34-46.

Moseley, G. L., Gallagher, L., \& Gallace, A. (2012) Neglect-like tactile dysfunction in chronic back pain. Neurology, 79, 327-332.

Mouraux, A., Diukova, A., Lee, M. C., Wise, R. G., \& Iannetti, G. D. (2011) A multisensory investigation of the functional significance of the "pain matrix". NeuroImage, 54, 2237-2249.

Peters, M. L, Vlaeyen, J. W. S., \& Kunnen, A. M. W. (2002) Is pain-related fear a predictor of somatosensory hypervigilance in chronic low back pain patients? Behaviour Research and Therapy, 7, 23-30.

Ploner, M., Pollok, B., \& Schnitzler, A. (2004) Pain facilitates tactile processing in human somatosensory cortices. Journal of Neurophysiology, 92, 1825-1829. 
Van Damme, S., \& Legrain, V. (2012) How efficient is the orienting of spatial attention to pain? An experimental investigation. Pain, 153, 1226-1231.

Van Damme, S., Crombez, G., \& Lorenz, J. (2007) Pain draws visual attention to its location: Experimental evidence for a threat-related bias. Journal of Pain, 8, 976-982.

Van Damme, S., Legrain, V., Vogt, J., \& Crombez, G. (2010) Keeping pain in mind: A motivational account of attention to pain. Neuroscience \& Biobehavioral Reviews, 34, 204-213.

Vanden Bulcke, C., Van Damme, S., Durnez, W., \& Crombez, G. (2013) The anticipation of pain at a specific location of the body prioritizes tactile stimuli at that location. Pain, 154, 1464-1468.

Van Hulle, L., Van Damme, S., Spence, C., Crombez, G., \& Gallace, A. (2013) Spatial attention modulates tactile change detection. Experimental Brain Research, 224, 295 302.

Vlaeyen, J. W. S., \& Linton, S. J. (2000) Fear-avoidance and its consequences in chronic musculoskeletal pain: A state of the art. Pain, 85, 317-332.

Weinstein, S. (1968) Intensive and extensive aspects of tactile sensitivity as a function of body part, sex and laterality. In D. R. Kenshalo (Ed.), The Skin Senses (pp. 195-200). Springfield, IL: Thomas. 


\section{Table 1}

Means, standard deviations and range of the tactor intensities (Power, in watts) at each body location. Numbers in brackets refer to the locations as depicted in Figure 1. Note that on the left lower arm (2) the reference stimulus was used which had the same intensity (0.04) in all participants.

\begin{tabular}{|c|c|c|c|c|}
\hline & $M$ & $S D$ & Min & $\operatorname{Max}$ \\
\hline Left upper arm (1) & 0.18 & 0.08 & 0.04 & 0.34 \\
\hline Left knee (3) & 0.32 & 0.15 & 0.11 & 0.83 \\
\hline Left ankle (4) & 0.20 & 0.09 & 0.04 & 0.34 \\
\hline Right upper arm (5) & 0.17 & 0.08 & 0.07 & 0.27 \\
\hline Right lower arm (6) & 0.06 & 0.04 & 0.02 & 0.21 \\
\hline Right knee (7) & 0.26 & 0.10 & 0.07 & 0.51 \\
\hline Right ankle (8) & 0.24 & 0.09 & 0.04 & 0.34 \\
\hline
\end{tabular}


Table 2

Means, standard deviations and ranges of the self-report items that were administered before (pre) and after (post) the experimental phase

\begin{tabular}{lccc}
\hline & $M$ & $S D$ & Range \\
\hline Painfulness ES - pre & 5.72 & 1.49 & $3-9$ \\
Unpleasantness ES - pre & 6.58 & 1.57 & $4-9$ \\
Painfulness ES - post & 6.29 & 1.58 & $3-9$ \\
Unpleasantness ES - post & 7.42 & 1.52 & $3-10$ \\
Expectation ES - post & 5.81 & 1.98 & $1-10$ \\
Fear for ES - post & 5.61 & 2.44 & $0-9$ \\
\hline
\end{tabular}



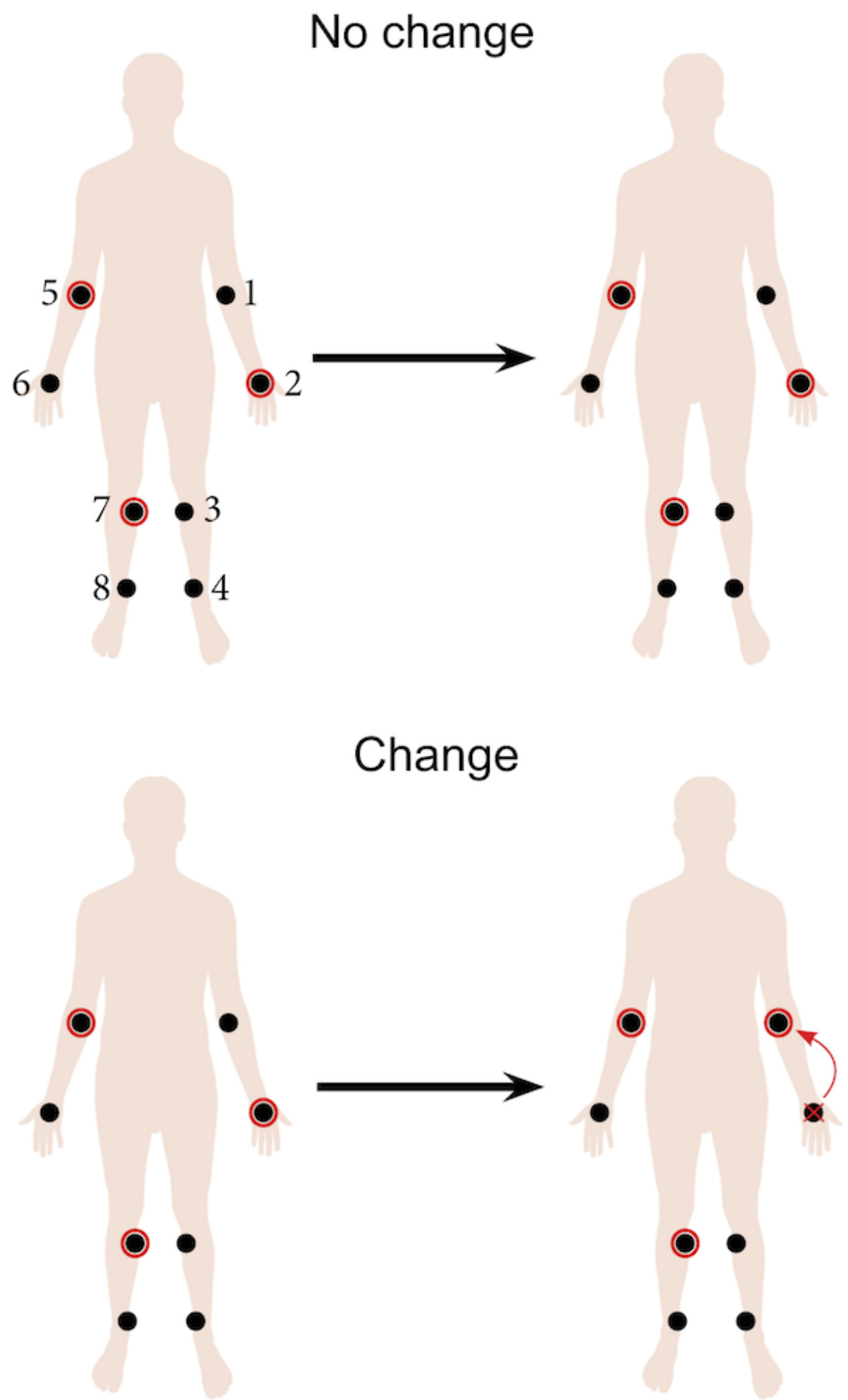

Figure 1. Example of a typical trial without change in the tactile pattern (top) and a trial with change (bottom). Tactor locations are numbered (see table 1 for mean intensities). 


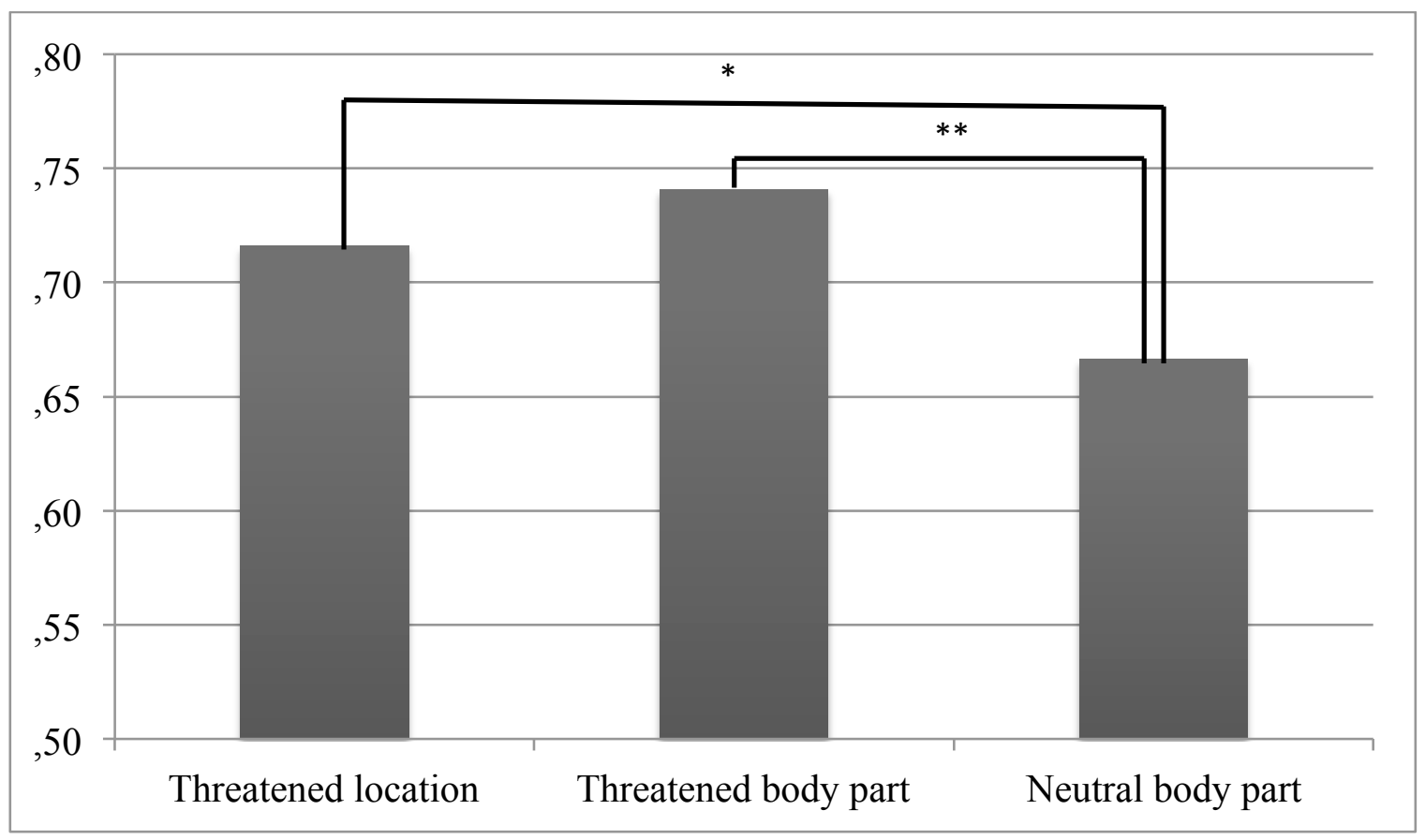

Figure 2. Mean proportion of correctly detected changes (Y-axis) as a function of Location (X-axis): Threatened location (a tactile stimulus emerged or disappeared at the exact pain location, i.e., the non-dominant lower arm), threatened body part (a tactile stimulus emerged or disappeared at the same body part as the pain location but at a different location, i.e., the non-dominant upper arm) and neutral body part (a tactile stimulus emerged or disappeared at a body part where no pain is expected). .[Note: $\left.{ }^{*} p<.05 ; * * p<.01\right]$ 